\title{
Sirtuin 1 regulates lipid metabolism associated with optic nerve regeneration
}

\author{
YAN ZHANG $^{1,2}$, HONGYANG LI ${ }^{1}$, YONGMEI CAO ${ }^{3}$, MAONIAN ZHANG $^{1}$ and SHIHUI WEI ${ }^{1}$ \\ ${ }^{1}$ Department of Ophthalmology, Chinese PLA General Hospital, Beijing 100085; \\ ${ }^{2}$ Department of Ophthalmology, The Military General Hospital of Beijing PLA, Beijing 100700; \\ ${ }^{3}$ Department of Ophthalmology, International Mongolion Hospital, Hohhot, Inner Mongolia 010065, P.R. China
}

Received November 14, 2014; Accepted August 14, 2015

DOI: $10.3892 / \mathrm{mmr} .2015 .4286$

\begin{abstract}
Injury to the optic nerve may lead to axonal degeneration, followed by the gradual death of retinal ganglion cells (RGCs), which results in irreversible vision loss. In the present study the mechanism of optic nerve injury, and the following regeneration and repair processes associated with sirtuin 1 (SIRT1)-regulated lipid metabolism were analyzed. In addition, the treatment of optic nerve injury using resveratrol was investigated. A rat model of optic nerve damage was prepared, and rats were divided into control, model and resveratrol groups. The model rats (with optic nerve damage) were treated with phosphate-buffered saline or resveratrol once a day for seven, 14 and 21 days. The rats were then sacrificed and the optic nerve was dissected. The expression levels of SIRT1, sterol regulatory element-binding protein 2 (SREBP-2) and 3-hydroxy-3-methylglutaryl-CoA reductase (HMGCR) mRNA in the optic nerve were measured by reverse transcription-quantitative polymerase chain reaction, and the protein expression of SIRT1, SREBP2 and HMGCR was evaluated by western blotting. In addition, the cholesterol level of the optic nerve was assessed. The retina was excised and the surviving RGCs were observed and counted. The morphology of the RGCs in the rat model of optic nerve injury changed; however, the degree of damage in rats treated with resveratrol was relatively small. The number of surviving RGCs and the cholesterol level in the RGCs from the model rats was observed to be restored by treatment with resveratrol following optic nerve damage. Additionally, the expression levels of SIRT1, SREBP-2 and HMGCR mRNA and protein were restored by resveratrol treatment in the rats with optic nerve damage. Thus, resveratrol reversed certain features of the optic nerve injury damage via SIRT1 and SREBP-2-associated signaling pathways and the downstream regulated gene, HMGCR. Furthermore,
\end{abstract}

Correspondence to: Professor Shihui Wei, Department of Ophthalmology, Chinese PLA General Hospital, 28 Fuxin Road, Beijing 100085, P.R. China

E-mail: weishihui1958@163.com

Key words: optic nerve damage, retinal ganglion cells, sirtuin 1, cholesterol regulatory element binding protein 2 , cholesterol synthesis resveratrol promoted cholesterol synthesis in, and repair of, RGCs. Therefore, SIRT1 may serve as a promising novel target for the treatment of optic nerve damage.

\section{Introduction}

The poor regenerative capacity of injured central nervous system (CNS) axons leads to permanent neurological deficits following brain, spinal cord or optic nerve lesions. Recent studies regarding the optic nerve revealed that stimulation of the cytokine or mammalian target of rapamycin signaling pathways potently enhances sprouting and regeneration of injured retinal ganglion cell (RGC) axons in adult mice, however, does not allow the majority of axons to reach their primary cerebral targets. Furthermore, recent studies have revealed axon navigation defects in the optic nerve and at the optic chiasm as a result of strong growth stimulation (1).

As with other CNS tracts in higher vertebrates, the optic nerve is unable to regenerate following traumatic injury. Optic nerve crush (ONC) is a popular model for investigating this phenomenon, and establishing novel regenerative strategies and potential treatment methods. The adeno-associated virus, serotype 2 was shown to display specific tropism to, and a high rate of infectivity for, RGCs; allowing researchers to target and manipulate specific intracellular signaling molecules involved in CNS neuron survival and axonal regeneration in vivo (2). The weak intrinsic capacity of adult neurons to reactivate a growth program following injury was proposed to be a significant impediment to axonal regrowth (3). Indeed, following birth, intrinsic growth repressors, for example, those of the Kruppel-like factor transcription factor family, are upregulated in the developing retina, while growth-inducing transcription factors are downregulated in RGCs and other CNS neurons (4). Similarly, the neuronal tissue environment is growth enhancing during development, and switches to being predominantly growth inhibitory in the adult brain and spinal cord $(5,6)$. The presence of growth-repressing molecules in the CNS tissue and within the neurons, as well as the lack of adequate stimulation and growth responses by adult neurons are currently proposed to be the predominant causes of axon regeneration failure (6).

SIRT1 belongs to a family of class III histone deacetylases, originally identified as significant to calorie restriction and longevity (7). SIRT1 activity is dependent on $\mathrm{NAD}^{+}$as a 
co-activator (8), and SIRT1 generally localizes to the nucleus, although it may translocate to the cytoplasm and catalyzes deacetylation of various protein targets in addition to histones (9). Numerous SIRT1 substrates are transcription factors, including peroxisome proliferator-activated receptor $\gamma$ coactivator $1 \alpha$, forkhead box $\mathrm{O} 3$ (FOXO), and hypoxia-inducible factor, whose activities are altered by SIRT1-mediated deacetylation, leading to altered expression levels of target genes (9-11). SIRT1 has been associated with various biologic processes, including oxidative stress, gene silencing and DNA repair, in addition to senescence, neurogenesis, circadian rhythms, neuroendocrine signals and dendritic branching, all of which affect the NS during normal physiologic functioning, as well as with aging and during pathologic processes (12). Within the brain, SIRT1 is crucial for normal cognitive function (13), and its activation protects against neurodegenerative diseases, including Parkinson's, Alzheimer's and Huntington's disease, as observed in animal models (14-16). In addition to its role in neurons, Sirtl contributes to blood vessel growth during development via regulation of Notch signaling (17) and FOXO1 (18). Chen et al (19) observed that SIRT1 is upregulated in RGCs in the vaso-obliteration zone of ischemic neuronal retinas, and conditional depletion of SIRT1 in retinal neurons significantly impaired vascular regrowth into the vascular zone and precipitated pathologic neovascularization in retinopathy. These results indicate that SIRT1 is a critical, stress-induced, metabolically-dependent protective mechanism in retinopathy (20).

In the current study the mechanism of optic nerve injury, and the subsequent regeneration and repair associated with SIRT1-regulated lipid metabolism was investigated. In addition, the efficacy of resveratrol treatment on optic nerve injury was assessed. It is hypothesized that a balanced growth stimulatory treatment, combined with guidance factors and suppression of local growth inhibitory factors, is required to obtain full regeneration of the optic nerve following injury.

\section{Materials and methods}

Animals. All animal procedures adhered strictly to the guidelines of the Association for Research in the Vision and Ophthalmology Statement on the Use of Animals in Ophthalmic and Visual Research, and were performed according to approved protocols by the Board of Animal Welfare at the Chinese PLA General Hospital (Beijing, China).

Adult male Sprague Dawley rats (n=70; weight, 280-320 g; (Sino-British SIPPR/BK Lab Animal Ltd., Shanghai, China) were used in the present study, and the results of eye and fundus examinations were normal. The rats were housed in a commercial cage. They were maintained under a 12-h light/dark cycle at an ambient temperature $\left(22 \pm 1^{\circ} \mathrm{C}\right)$ and given free access to food. Using the random number table method for grouping, 10 rats were assigned to the control group, while 30 rats formed the model group [treated with phosphate-buffered saline (PBS; Invitrogen Life Technologies, Inc., Carlsbad, CA, USA)] and 30 rats formed the Res group (treated with resveratrol; Sigma-Aldrich, St. Louis, MO, USA). Each of the model and Res groups were comprised of three subgroups $(n=10)$ of rats that were treated for either seven, 14 or 21 days.
Model preparation and treatment process. Following intraperitoneal injection of $50 \mathrm{mg} / \mathrm{kg}$ ketamine (Sigma-Aldrich) to anesthetize the rats, the optic nerve was fully exposed. Then reverse tweezers with an optic ball (cross-locking tweezers, bent $\&$ straight reverse action, stainless steel, 4-3/4" 2pc; size, $0.5-1.0 \mathrm{~mm}$ ) were used to clamp the optic nerve and cause incomplete injury of rats. In the resveratrol-treated group following postoperative injury, an intraperitoneal injection of $30 \mathrm{mg} / \mathrm{kg}$ resveratrol was administered every $12 \mathrm{~h}$. The same protocol was followed in the control group, however, the intraperitoneal injection of resveratrol was replaced with PBS $(2.5 \mu \mathrm{l} / \mathrm{g})$. The rats were sacrificed seven, 14 or 21 days after treatment, and the optic nerves and retinas were excised.

Retina dissection, staining and imaging. The rats were anesthetized with Avertin (0.2 ml/10 g; Sigma-Aldrich) and sacrificed with $\mathrm{CO}_{2}$. The eyes were enucleated, fixed in $4 \%$ paraformaldehyde (Novus Biologicals, LLC, Littleton, CO, USA) in PBS for $1 \mathrm{~h}$ and dissected to isolate the retina. The retinas without retina pigment epithelium were rapidly ( $<5 \mathrm{~min}$ ) dissected from the eyeball on ice, according to the method of Glowinski and lverse (1). Each sample was immediately labeled and then flash-frozen in liquid nitrogen and stored at $-80^{\circ} \mathrm{C}$ for further analysis. The retinas were subsequently stained overnight with Isolectin GS-IB ${ }_{4}$ from Griffonia simplicifolia, Alexa Fluor ${ }^{\circledR} 594$ Conjugate (dilution, 1:100; Invitrogen Life Technologies) in PBS with $1 \mathrm{mM} \mathrm{CaCl}_{2}$ to visualize the blood vessels. After 2-h washes in PBS, the retinas were whole-mounted (photoreceptor side down) onto Superfrost ${ }^{\mathrm{TM}}$ Plus microscope slides (Thermo Fisher Scientific, Inc., Waltham, MA, USA) using SlowFade Antifade reagent (Invitrogen Life Technologies). Whole-mounted retinas were imaged using an AxioObserver.Z1 microscope (magnification, x5; Carls Zeiss AG, Oberkochen, Germany) and merged using AxioVision 4.6.3.0 software (Carl Zeiss AG) to produce images of the entire retinal vasculature. Estimates of RGC counts were obtained using a previously published model (20), which combines estimates of RGC numbers (from standard automated perimetry sensitivity thresholds and retinal nerve fiber layer thickness measurements) with spectral domain optical coherence tomography.

Reverse transcription-quantitative polymerase chain reaction $(R T-q P C R)$. Total RNA was prepared from retinal cells using the RNeasy Mini kit (Qiagen, Inc., Valencia, CA, USA) and subjected to RT by SuperScriptH III reverse transcriptase (Invitrogen Life Technologies) according to the manufacturer's instructions. A hot-start at $95^{\circ} \mathrm{C}$ for $5 \mathrm{~min}$ was followed by 40 cycles of denaturation at $95^{\circ} \mathrm{C}$ for $15 \mathrm{sec}$, annealing of the primers at $60^{\circ} \mathrm{C}$ for $30 \mathrm{sec}$ and elongation at $72^{\circ} \mathrm{C}$ for $30 \mathrm{sec}$, using an ABI 7500 Fast Real-Time PCR system (Invitrogen Life Technologies). Phire Hot Start II DNA Polymerase (Thermo Fisher Scientific, Inc.) incorporates a dsDNA-binding domain that allows short extension times (10-15 sec/kb), helps improve yields, and can increase the fidelity by two-fold compared to that of Taq DNA polymerase. In addition, the unique hot start technology allows complete re-activation of the enzyme in 'zero-time' at standard cycling temperatures. This combination of features makes Phire Hot Start II DNA Polymerase an ideal solution for routine and high-throughput PCR applications. 


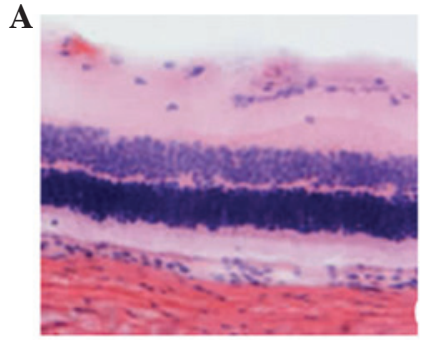

$\mathbf{E}$

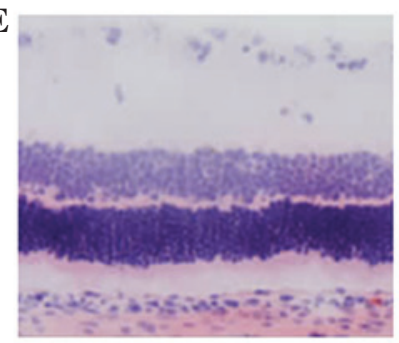

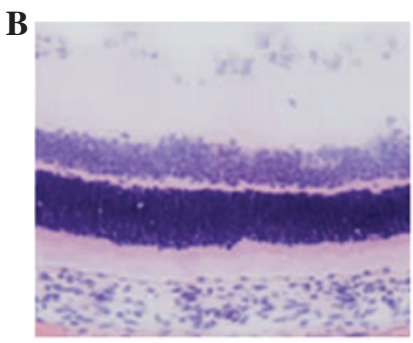

F

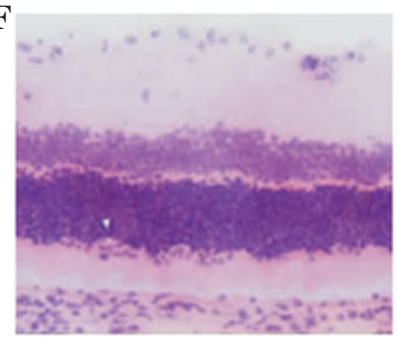

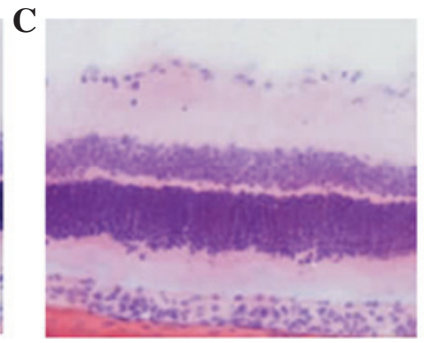
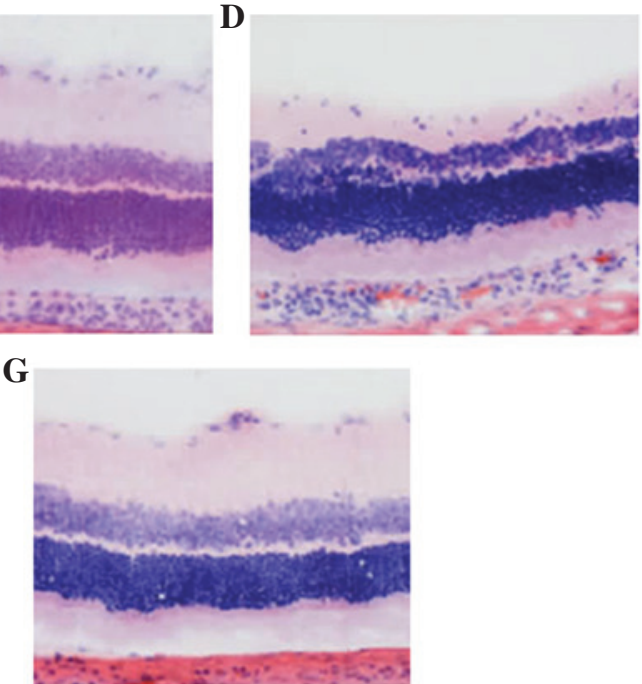

Figure 1. Morphological changes in the retina (magnification, $\mathrm{x} 400$; hematoxylin and eosin staining). (A) In the normal control rats, the ganglial cell layer as well as the inner or outer nuclear layers of the retinas were dense and arranged in parallel from the exterior to the interior. (B) Seven days after optic nerve injury, the cell nuclei of the ganglion cell layer exhibited a scattered distribution, volume shrinkage, and patterns of chromatin margination and condensation were observed. The inner and outer nuclear layers were thinning. (C) At 14 days after optic nerve injury, the cell nuclei count in the ganglion cell layer decreased markedly and demonstrated a sparse arrangement. A greater number of condensed nuclei was observed. (D) After 21 days, the cell count in each layer had decreased and ganglial cells rarely contained nuclei. In addition, chromatin margination, cavitation of ganglion cells and infiltration of glial cells was apparent. The cell count of the inner and outer nuclear layer decreased. The inner and outer nuclear layers were thinning, and the cell arrangement was disordered. (E) No marked changes were apparent in the rats that were treated with resveratrol. (F) The quantity of large and stained gangliocytic nuclei decreased and chromatin margination was observed in the rats treated with resveratrol. (G) The extent of damage in rats treated with resveratrol was relatively small.

The primer sequences used were as follows: Upstream, 5'-TGTACGACGACGAAGACGACGAC-3' and downstream, 5'-GGTTATCTCGGTACCCAATCG-3' for SIRT1; upstream, 5'-GCTGGCTCACCTTTCCAG-3' and downstream, 5'-GGCCTCAGTTATTCTGTCTTGC-3' for SREBP2; and upstream, 5'-CTTGAAGTTCATGGTGATCAGTG-3' and downstream, 5'-CCATTTTCAACTGGCAGGA-3' for HMGCR. Data were normalized to GAPDH and represented as the average value of three independent duplicate experiments.

Western blot analysis. Retinal cell lysates were prepared from cells using a lysis buffer [50 mM Tris ( $\mathrm{pH} 8), 150 \mathrm{mM}$ $\mathrm{NaCl}, 0.02 \% \mathrm{NaN}_{3}, 0.1 \% \mathrm{SDS}, 1 \% \mathrm{NP}-40$ and $0.5 \%$ sodium deoxycholate; Upstate Biotechnology, Lake Placid, NY, USA] containing $1 \mathrm{mM}$ phenylmethylsulfonyl fluoride (Sigma-Aldrich) and protease inhibitor cocktail (Roche Diagnostics, Indianapolis, IN, USA). The protein concentration was determined by Bradford assay using the Coomassie Plus Protein Reagent (Invitrogen Life Technologies) and western blot analysis was performed using the Novex ${ }^{\circledR}$ system (Invitrogen Life Technologies).

Blots were incubated with primary antibodies anti-SIRT1 [cat no. sc-15404; H-300; rabbit polyclonal immunoglobulin (Ig)G], anti-SREBP-2 (cat no. sc-13552; 1C6; mouse monoclonal $\mathrm{IgG}_{1}$ ) or anti-HMGCR (cat no. sc-27578; C-18; goat polyclonal $\mathrm{IgG}$ ) (all from Santa Cruz Biotechnology, Inc., Dallas, TX, USA) at $4^{\circ} \mathrm{C}$ overnight (dilution, 1:1,000) and washed with PBS three times. Subsequently, blots were incubated with horseradish peroxidase-conjugated secondary antibodies (GE Healthcare Bio-Sciences, Pittsburgh, PA, USA) at room temperature for $1 \mathrm{~h}$ (dilution, 1:5,000) and washed with PBS three times. Protein bands were detected using Enhanced
Chemiluminescence Western Blotting Detection Reagent (GE Healthcare Bio-Sciences).

Cholesterol level quantification in the optic nerve. The cholesterol level was determined in the optic nerve tissues using the Cholesterol/Cholesteryl Ester Detection kit (Abcam, Cambridge, MA, USA) by oxidase-peroxidase enzymatic assay according to the manufacturer's instructions. Briefly, $10 \mu \mathrm{l}$ suspension liquid of optic nerve tissues and $1 \mathrm{ml}$ working fluid (oxidase peroxidase enzyme in cholesterol assay buffer) were incubated at $37^{\circ} \mathrm{C}$ in a water bath for $10 \mathrm{~min}$, and then the absorbance value of cholesterol standard pipe (A) and samples (B) were determined under the spectrophotometer (Mithras LB 940; Berthold Technologies, Bad Wildbad, Germany)at a wavelength of $500 \mathrm{~nm}$. The following formula was used to calculate the content of cholesterol in every $10 \mathrm{~g}$ of tissue protein: Cholesterol level $(\mathrm{mg} / 10 \mathrm{~g}$ tissue protein $)=[(\mathrm{A} / \mathrm{B}) \mathrm{x}$ concentration of standard fluid x 150/weight of sample] x 10 .

Statistical analysis. Continuous normally distributed variables were represented graphically as the mean \pm standard deviation. For statistical comparison of quantitative data between groups, analysis of variance (ANOVA) or a two-tailed Student's t-test was performed. To determine differences between groups that were not normally distributed, medians were compared using Kruskal-Wallis ANOVA. The $\chi^{2}$ test was used when necessary for qualitative data. The degree of association between variables was assessed using Spearman's non-parametric correlation. All statistical analyses were conducted using SPSS software version 13.0 (SPSS, Inc., Chicago, IL, USA) and $\mathrm{P}<0.05$ was considered to indicate a statistically significant difference. 
Table I. Number of RGCs at each time point.

\begin{tabular}{llccc}
\hline & & \multicolumn{3}{c}{ Number of RGCs } \\
\cline { 3 - 5 } Group & $\mathrm{n}$ & 7 days & 14 days & 21 days \\
\hline Control & 10 & $33.18 \pm 5.02$ & $32.47 \pm 4.38$ & $34.65 \pm 5.18$ \\
Resveratrol & 30 & $29.46 \pm 3.28^{\mathrm{a}}$ & $25.19 \pm 2.54^{\mathrm{a}}$ & $22.66 \pm 1.87^{\mathrm{a}}$ \\
Model & 30 & $17.31 \pm 3.18$ & $14.55 \pm 2.28$ & $13.62 \pm 2.88$
\end{tabular}

${ }^{\mathrm{a}} \mathrm{P}<0.01$ vs. control group. RGC, retinal ganglion cell.

\section{Results}

Effective establishment of the rat model of optic nerve injury. Following excision, the rats' wounds healed well and there was no inflammatory response. The corneas were transparent and there were no cases of traumatic cataract. Furthermore, no vitreous inflammatory reaction was observed and there was no blood in the vitreous cavity, retinal bleeding or detachment of the retina as a result of the surgery. Mydriasis was apparent following surgery (diameter, of 2-4 mm), indicated by a direct (-) and indirect light reflex (+), which indicated relative afferent pupillary defect. These evaluation indexes indicated the successful creation of the rat model of optic nerve injury.

Morphological changes of the retina. The ganglion cell layer, and inner and outer nuclear layers of the retina of the normal control rats, from outside to inside, were dense and arranged in parallel. Seven days after optic nerve injury, the cell nuclei of the ganglion cell layer exhibited scattered distribution, shrinkage of the nuclei volume, and chromatin margination and condensation. The inner and outer nuclear layers were thinning and the cell arrangement was disordered. However, no marked changes were observed in the rats treated with resveratrol. At 14 days after optic nerve injury, the cell nuclei count in the ganglion cell layer decreased markedly, and demonstrated a sparse arrangement and increased nuclear condensation. The inner and outer nuclear layers had become thinner. In the rats treated with resveratrol, the number of large and stained nuclei of the gangliocytes decreased, while the nuclei of the gangliocytes exhibited slight changes in size or number. After 21 days, the cell count in each layer decreased and few nuclei were observed in the gangliocytes. Chromatin margination, cavitation of the RGCs and glial cell infiltration were observed. The cell count in the inner and outer nuclear layer decreased. However, the extent of damage was relatively small in the resveratrol-treated rats (Fig. 1).

Resveratrol restores $R G C$ number. The number of surviving RGCs in the rat models significantly reduced, in a time-dependent manner, when compared with those in the control group at each time point $(\mathrm{P}<0.01)$. In the resveratrol-treated group, the number of surviving RGCs decreased marginally at each time point, in a time-dependent manner, when compared with the control group $(\mathrm{P}<0.01)$. The differences between the resveratrol group and the control group were statistically significant

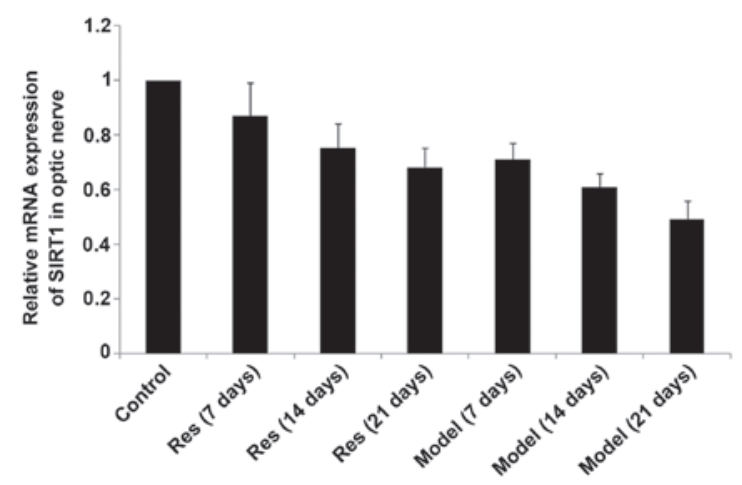

Figure 2. Expression level of SIRT1 mRNA in the optic nerve. The expression level of SIRT1 mRNA in the rat model of optic nerve injury reduced significantly and in a time-dependent manner at each time point when compared with the control group $(\mathrm{P}<0.01)$. The expression level of SIRT1 mRNA in the Res group decrease marginally, and in a time-dependent manner, at each time point when compared with the control group $(\mathrm{P}<0.05)$. The difference between the Res group and the model group at each time point was statistically significant $(\mathrm{P}<0.01)$. Thus, the expression level of SIRT1 mRNA may be restored by treatment with Res in rats with optic nerve damage. SIRT1, sirtuin 1; Res, resveratrol.

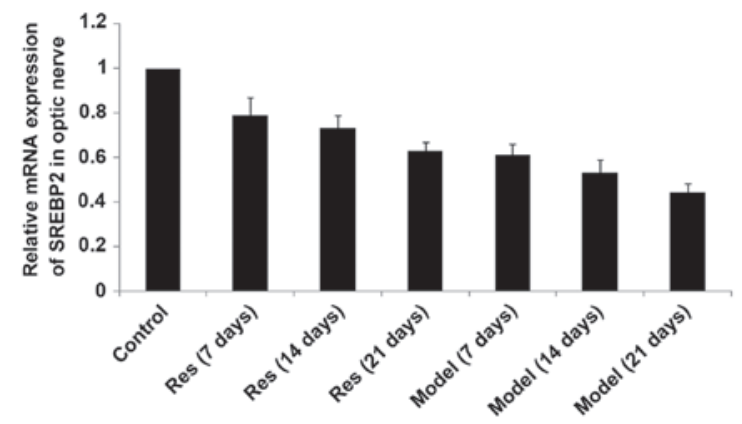

Figure 3. Expression level of SREBP-2 mRNA in the optic nerve. The expression level of SREBP-2 mRNA in the rat model of optic nerve injury reduced significantly and in a time-dependent manner at each time point when compared with the control group $(\mathrm{P}<0.01)$. The expression level of SREBP-2 mRNA in the Res group decrease marginally, and in a time-dependent manner, at each time point when compared with the control group $(\mathrm{P}<0.05)$. The difference between the Res group and the model group at each time point was statistically significant $(\mathrm{P}<0.01)$. Thus, the expression level of SREBP-2 mRNA may be restored by treatment with Res in rats with optic nerve damage. SREBP-2, sterol regulatory element-binding protein 2; Res, resveratrol.

at each time point $(\mathrm{P}<0.01$; Table I). This indicated that the number of surviving RGCs in rats with optic nerve damage was restored by treatment with resveratrol. 
Table II. Cholesterol levels in RGCs.

Cholesterol levels in RGCs (mg/10 g tissue protein)

\begin{tabular}{lcccc}
\cline { 3 - 4 } Group & $\mathrm{n}$ & 7 days & 14 days & 21 days \\
\hline Control & 10 & $102.26 \pm 4.23$ & $108.35 \pm 7.33$ & $110.79 \pm 6.25$ \\
Resveratrol & 30 & $87.54 \pm 8.63^{\mathrm{a}}$ & $72.15 \pm 6.38^{\mathrm{a}}$ & $64.38 \pm 5.14^{\mathrm{a}}$ \\
Model & 30 & $54.21 \pm 4.67^{\mathrm{b}, \mathrm{c}}$ & $43.59 \pm 3.96^{\mathrm{b}, \mathrm{c}}$ & $32.65 \pm 3.12^{\mathrm{b}, \mathrm{c}}$
\end{tabular}

${ }^{\mathrm{a}} \mathrm{P}<0.01$ vs. control group; ${ }^{\mathrm{b}}<0.01$ control vs. model group; ${ }^{\mathrm{c}} \mathrm{P}<0.01$ resveratrol vs. model group. RGCs, retinal ganglion cells.

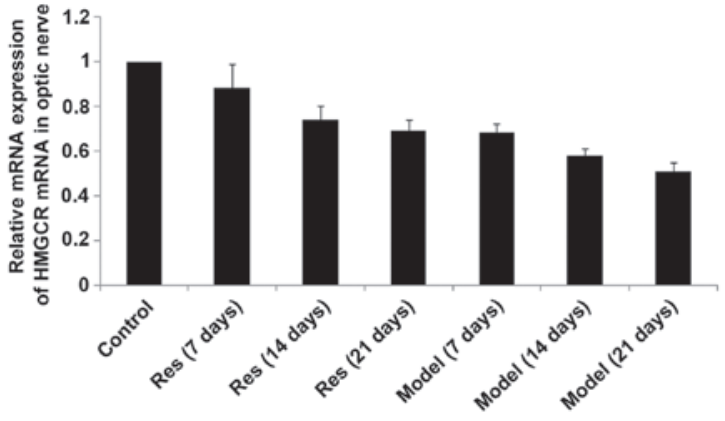

Figure 4. Expression level of HMGCR mRNA in the optic nerve. The expression level of HMGCR mRNA in the rat model of optic nerve injury reduced significantly and in a time-dependent manner at each time point when compared with the control group $(\mathrm{P}<0.01)$. The expression level of HMGCR mRNA in the Res group decrease marginally, and in a time-dependent manner, at each time point when compared with the control group $(\mathrm{P}<0.05)$. The difference between the Res group and the model group at each time point was statistically significant $(\mathrm{P}<0.01)$. Thus, the expression level of HMGCR mRNA may be restored by treatment with Res in rats with optic nerve damage. HMGCR, 3-hydroxy-3-methylglutaryl-CoA reductase; Res, resveratrol.

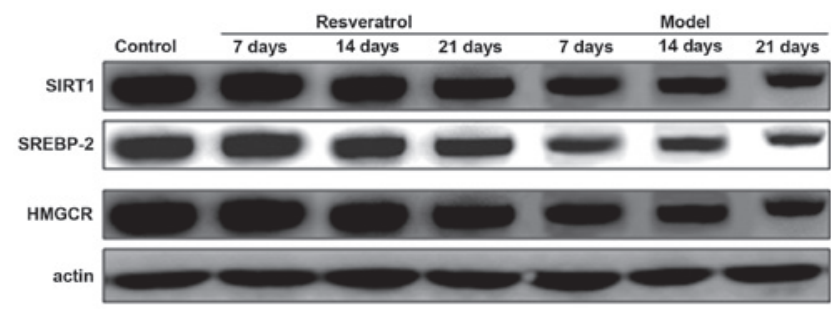

Figure 5. Expression levels of the SIRT1, SREBP-2 and HMGCR proteins in the rat model of optic nerve injury. The expression levels of the SIRT1 SREBP-2 and HMGCR proteins in the rat model group reduced significantly and in a time-dependent manner at each time point when compared with the control group $(\mathrm{P}<0.01)$. The expression levels of the SIRT1, SREBP-2 and HMGCR proteins in the Res group decreased marginally and in a time-dependent manner at each time point when compared with the control group $(\mathrm{P}<0.05)$. The difference between the Res group and the model group at each time point was statistically significant $(\mathrm{P}<0.01)$. Thus, the expression level of the SIRT1, SREBP-2 and HMGCR proteins may be restored by treatment with Res in rats with optic nerve damage. SIRT1, sirtuin 1; SREBP-2, sterol regulatory element-binding protein 2; HMGCR, 3-hydroxy-3-methylglutaryl-CoA reductase; Res, resveratrol.

Cholesterol levels in $R G C s$ are restored by resveratrol treatment. The levels of cholesterol in the RGCs of the model rats reduced significantly and in a time-dependent manner when compared with those of the control group at each time point
$(\mathrm{P}<0.01)$. The cholesterol levels of the rats in the resveratrol group decreased marginally at each time point and in a time-dependent manner compared with the control group $(\mathrm{P}<0.05)$. The differences between the resveratrol group and the model group were statistically significant at each time point $(\mathrm{P}<0.01)$. Thus, cholesterol levels in the RGCs of rat models of optic nerve damage were restored by treatment with resveratrol (Table II).

SIRT1, SREBP-2 and HMGCR mRNA levels decrease in rat models of optic nerve injury. The expression levels of SIRT1, SREBP-2 and HMGCR mRNA (Figs. 2-4, respectively) in the rat models of optic nerve injury reduced significantly, and in a time-dependent manner, at each time point when compared with the control group $(\mathrm{P}<0.01)$. The expression levels of SIRT1, SREBP-2 and HMGCR mRNA (Figs. 2-4, respectively) in the resveratrol group decreased marginally, and in a time-dependent manner, at each time point when compared with the normal group $(\mathrm{P}<0.05)$. The differences between the resveratrol group and the model group were statistically significant at each time point $(\mathrm{P}<0.01)$. Therefore, treatment with resveratrol restored the expression levels of SIRT1, SREBP-2 and HMGCR mRNA (Figs. 2-4, respectively) in rat models of optic nerve damage.

Levels of SIRT1, SREBP-2 and HMGCR protein decrease in rat models of optic nerve injury. The expression levels of SIRT1, SREBP-2 and HMGCR proteins in the rat models of optic nerve injury reduced significantly, and in a time-dependent manner, at each time point when compared with the control group $(\mathrm{P}<0.01)$. The expression levels of SIRT1, SREBP-2 and HMGCR proteins in the resveratrol group decreased marginally at each time point and in a time-dependent manner compared with the normal group $(\mathrm{P}<0.05)$. The differences between the resveratrol group and the model group at each time point were statistically significant $(\mathrm{P}<0.01)$. The findings demonstrate that the expression level of SIRT1, SREBP-2 and HMGCR proteins in rat models of optic nerve damage were restored by treatment with resveratrol (Fig. 5).

\section{Discussion}

The retina and optic nerve form part of the CNS. Since CNS damage is irreversible, injuries to the optic nerve, such as those caused by trauma, inflammation and ocular hypertension, lead to RGC loss, which results in permanent loss of vision 
or blindness. Currently, and to the best of our knowledge, no therapeutic agent has been developed for the effective treatment of optic nerve injury. Therefore, a neuroprotective remedy that delays or prevents RGC loss following optic nerve injury is required (21).

Lipidomics, a subgroup within the field of metabolomics, presents two areas of interest. Architecture/membrane lipidomics describes the comprehensive and quantitative nature of membrane lipid constituents, while mediator lipidomics includes the structural characterization and quantification of bioactive lipid species (8). The lipid composition of the membranes of numerous tissues and cell types has been well established (13), however, little is known regarding the distribution of lipids within these membranes. One specific class of lipid molecules, the docosanoids, appears to be significant in retinal structure and function, as well as being involved in the orchestration of homeostasis, anti-inflammation, inflammatory resolution, and cell survival bioactivity $(22,23)$. Previous studies have described the following observations: (i) Docosahexaenoic acid (DHA) from the liver is targeted to the brain and retina, where it is actively retained and protected from peroxidation (23-25). When lipid peroxidation is triggered in animal models of retinal degeneration, alterations in photoreceptor function, damage and cell death are observed. (ii) The blood concentration of DHA is decreased in various forms of retinitis pigmentosa and in Usher syndrome, indicating that decreased DHA supply may cause photoreceptor impairment $(22,26)$ and when animal models overexpress rhodopsin mutations that are homologous to human retinitis pigmentosa, the photoreceptors contain a reduced quantity of DHA (27). However, while these observations may suggest that reduced retinal DHA is associated with photoreceptor dysfunction, a reduction in DHA may, instead, be an adaptive response to metabolic stress caused by the mutation $(27,28)$. Furthermore, the association between circulating DHA, retinal DHA and pathology has not been conclusively demonstrated, and human data has been obtained that does not reveal a positive association between circulating and retinal DHA $(29,30)$ or between circulating DHA and age-related macular degeneration (31). Therefore, it is important to note that the association between decreased DHA availability, disease initiation and progression of retinal degeneration remains unclear. (iii) Finally, in constant light-mediated retinal degeneration, photoreceptor DHA levels are reduced, and rats reared in bright cyclic light were shown to be protected from DHA loss and degeneration, which indicates a mechanism of adaptation and/or a plasticity response (31). DHA, in conjunction with pigment epithelial-derived factor, triggers early synthesis of neuroprotectin D1, which is neuroprotective, and promotes regeneration of corneal nerves and restores corneal sensitivity.

In the present study, the neuroprotective effect of the agonist for SIRT1 was examined, in addition, the efficacy of resveratrol treatment on RGCs was analyzed and the role of cholesterol synthesis was investigated following ONC injury in rats. In the control group, the ganglion cell layers of the retinas, including inner and outer nuclear layers, were dense and arranged in parallel. After optic nerve injury, they exhibited a scattered distribution; the nuclei changed, the inner and outer nuclear layer were thinner and a disordered arrangement of cells was observed. Of note, there was significant improvement in rats treated with resveratrol, with the degree of damage being markedly attenuated by resveratrol in a concentration-dependent manner. The retinas were damaged in model rats with optic nerve injury, while the degree of damage in rats treated with resveratrol was relatively low. The number of surviving RGCs and the content of cholesterol in RGCs of model rats was restored by resveratrol. Resveratrol treatment also restored the mRNA and protein expression levels of SIRT1, SREBP2 and HMGCR.

In conclusion, treatment with resveratrol represents a novel approach for regenerating the optic nerve, as well as a potential treatment for prevention of severe injury following surgery or disease of the ocular surface. Notably, mediator lipidomic characterization of signaling mechanisms, triggered by stress and cellular damage that upregulate neuroprotective events leading to nerve regeneration, may provide unique pharmacological targets for numerous neuronal and ocular degenerative diseases.

\section{Acknowledgements}

The present study was supported the National Natural Science Foundation of China (grant no. 30600524).

\section{References}

1. Pernet V and Schwab ME: Lost in the jungle: New hurdles for optic nerve axon regeneration. Trends Neurosci 37: 381-387, 2014.

2. Pernet V, Hauswirth WW and Di Polo A: Extracellular signal-regulated kinase 1/2 mediates survival, but not axon regeneration, of adult injured central nervous system neurons in vivo. J Neurochem 93: 72-83, 2005.

3. Goldberg JL, Klassen MP, Hua Y and Barres BA: Amacrine-signaled loss of intrinsic axon growth ability by retinal ganglion cells. Science 296: 1860-1864, 2002.

4. Moore DL, Blackmore MG, Hu Y, Kaestner KH, Bixby JL, Lemmon VP and Goldberg JL: KLF family members regulate intrinsic axon regeneration ability. Science 326: 298-301, 2009.

5. Schwab ME: Functions of Nogo proteins and their receptors in the nervous system. Nat Rev Neurosci 11: 799-811, 2010.

6. Yiu $\mathrm{G}$ and He Z: Glial inhibition of $\mathrm{CNS}$ axon regeneration. Nat Rev Neurosci 7: 617-627, 2006.

7. Guarente L and Franklin H: Epstein lecture: Sirtuins, aging and medicine. N Engl J Med 364: 2235-2244, 2011.

8. Canto C, Gerhart-Hines Z, Feige JN, Lagouge M, Noriega L, Milne JC, Elliott PJ, Puigserver P and Auwerx J: AMPK regulates energy expenditure by modulating NAD+ metabolism and SIRT1 activity. Nature 458: 1056-1060, 2009.

9. Brunet A, Sweeney LB, Sturgill JF, Chua KF, Greer PL, Lin Y, Tran H, Ross SE, Mostoslavsky R, Cohen HY, et al: Stressdependent regulation of FOXO transcription factors by the SIRT1 deacetylase. Science 303: 2011-2015, 2004.

10. Rodgers JT, Lerin C, Haas W, Gygi SP, Spiegelman BM and Puigserver P: Nutrient control of glucose homeostasis through a complex of PGC-1alpha and SIRT1. Nature 434: 113-118, 2005.

11. Dioum EM, Chen R, Alexander MS, Zhang Q, Hogg RT, Gerard RD and Garcia JA: Regulation of hypoxia-inducible factor 2alpha signaling by the stress-responsive deacetylase sirtuin 1. Science 324: 1289-1293, 2009.

12. Michan S: Acetylome regulation by sirtuins in the brain: From normal physiology to aging and pathology. Curr Pharm Des 19: 6823-6838, 2013.

13. Michan S, Li Y, Chou MM, Parrella E, Ge H, Long JM, Allard JS, Lewis K, Miller M, Xu W, et al: SIRT1 is essential for normal cognitive function and synaptic plasticity. J Neurosci 30: 9695-9707, 2010.

14. Donmez G, Wang D, Cohen DE and Guarente L: SIRT1 suppresses beta-amyloid production by activating the alpha-secretase gene ADAM10. Cell 142: 320-332, 2010. 
15. Jiang M, Wang J, Fu J, Du L, Jeong H, West T, Xiang L, Peng Q, Hou Z, Cai H, et al: Neuroprotective role of Sirtl in mammalian models of Huntington's disease through activation of multiple Sirt1 targets. Nat Med 18: 153-158, 2011.

16. Donmez G, Arun A, Chung CY, McLean PJ, Lindquist S and Guarente L: SIRT1 protects against $\alpha$-synuclein aggregation by activating molecular chaperones. J Neurosci 32: 124-132, 2012.

17. Guarani V, Deflorian G, Franco CA, Krüger M, Phng LK, Bentley K, Toussaint L, Dequiedt F, Mostoslavsky R, Schmidt MH, et al: Acetylation-dependent regulation of endothelial Notch signalling by the SIRT1 deacetylase. Nature 473: 234-238, 2011.

18. Potente M, Ghaeni L, Baldessari D, Mostoslavsky R, Rossig L, Dequiedt F, Haendeler J, Mione M, Dejana E, Alt FW, et al: SIRT1 controls endothelial angiogenic functions during vascular growth. Genes Dev 21: 2644-2658, 2007.

19. Chen J, Michan S, Juan AM, Hurst CG, Hatton CJ, Pei DT, Joyal JS, Evans LP, Cui Z, Stahl A, et al: Neuronal sirtuin1 mediates retinal vascular regeneration in oxygen-induced ischemic retinopathy. Angiogenesis 16: 985-992, 2013.

20. Michan S, Juan AM, Hurst CG, Cui Z, Evans LP, Hatton CJ, Pei DT, Ju M, Sinclair DA, Smith LE and Chen J: Sirtuin1 over-expression does not impact retinal vascular and neuronal degeneration in a mouse model of oxygen-induced retinopathy. PLoS One 9: e85031, 2014.

21. Jiao X, Peng Y and Yang L: Minocycline protects retinal ganglion cells after optic nerve crush injury in mice by delaying autophagy and upregulating nuclear factor- $\mathrm{KB} 2$. Chin Med J (Engl) 127 : 1749-1754, 2014

22. Bazan NG: Homeostatic regulation of photoreceptor cell integrity: Significance of the potent mediator neuroprotectin D1 biosynthesized from docosahexaenoic acid: The proctor lecture. Invest Ophthalmol Vis Sci 48: 4866-4881, 2007.

23. Bazan NG, Birkle DL and Reddy TS: Biochemical and nutritional aspects of the metabolism of polyunsaturated fatty acids and phospholipids in experimental models of retinal degeneration. In: Retinal Degeneration: Experimental and Clinical Studies. LaVail MM, Hollyfield JG and Anderson RE (eds). Vol 2. A.R. Liss, New York, NY, pp159-187, 1985.
24. Scott BL and Bazan NG: Membrane docosahexaenoate is supplied to the developing brain and retina by the liver. Proc Natl Acad Sci USA 86: 2903-2907, 1989.

25. Gordon WC, Rodriguez de Turco EB and Bazan NG: Retinal pigment epithelial cells play a central role in the conservation of docosahexaenoic acid by photoreceptor cells after shedding and phagocytosis. Curr Eye Res 11: 73-83, 1992.

26. Hoffman DR, Boettcher JA and Diersen-Schade DA: Toward optimizing vision and cognition in term infants by dietary docosahexaenoic and arachidonic acid supplementation: A review of randomized controlled trials. Prostaglandins Leukot Essent Fatty Acids 81: 151-158, 2009.

27. Anderson RE, Maude MB and Bok D: Low docosahexaenoic acid levels in rod outer segment membranes of mice with rds/peripherin and $\mathrm{P} 216 \mathrm{~L}$ peripherin mutations. Invest Ophthalmol Vis Sci 42: 1715-1720, 2001.

28. Gordon WC and Bazan NG: Mediator lipidomics in ophthalmology: Targets for modulation in inflammation, neuroprotection and nerve regeneration. Curr Eye Res 38: 995-1005, 2013.

29. Bretillon L, Thuret G, Grégoire S, Acar N, Joffre C, Bron AM, Gain P and Creuzot-Garcher CP: Lipid and fatty acid profile of the retina, retinal pigment epithelium/choroid and the lacrimal gland and associations with adipose tissue fatty acids in human subjects. Exp Eye Res 87: 521-528, 2008.

30. Acar N, Berdeaux O, Grégoire S, Cabaret S, Martine L, Gain P, Thuret G, Creuzot-Garcher CP, Bron AM and Bretillon L: Lipid composition of the human eye: Are red blood cells a good mirror of retinal and optic nerve fatty acids? PLoS One 7: e35102, 2012.

31. Kabasawa S, Mori K, Horie-Inoue K, Gehlbach PL, Inoue S, Awata T, Katayama S and Yoneya S: Associations of cigarette smoking but not serum fatty acids with age-related macular degeneration in a Japanese population. Ophthalmology 118: 1082-1088, 2011 